\title{
Vancomycin-resistant Enterococcus bacteremia in a child with acute myeloid leukemia: Successful treatment with daptomycin
}

\author{
Ayşe Büyükcam ${ }^{a}$, M.D., Eda Karadağ Öncel ${ }^{a}$, M.D., Yasemin Özsürekçia , M.D., Prof. Ali B.Cengiz , M.D., \\ Assoc. Prof. Barış Kuşkonmaz , M.D., and Prof. Banu Sancak ${ }^{c}$, M.D.
}

\begin{abstract}
Multiple-drug-resistant enterococcal infections can be a serious problem in pediatric patients particularly concomitance with severe underlying diseases and lead to significant morbidity and mortality. The treatment options in children are limited compared with adults. We report a 3-year old-boy with acute myeloid leukemia (AML)-M7 and vancomycin-resistant enterococcus bacteremia successfully treated with daptomycin. Daptomycin may be an alternative therapy for VRE infections in children; more studies are needed for extended usage. Key words: vancomycin resistant Enterococci, child, daptomycin, linezolid.
\end{abstract}

http:/ /dx.doi.org/10.5546/aap.2016.eng.e432

\section{BACKGROUND}

Vancomycin-resistant Enterococcus (VRE) infections is a global clinical problem at the hospital settings and can cause infective endocarditis, catheter-related bloodstream infections, urinary tract infections and bacteremia of unknown source. VRE bacteremia is a major cause of nosocomial infections with a significant morbidity and mortality especially in children with underlying severe chronic diseases. ${ }^{1-3}$ Daptomycin is a cyclic lipopeptide antimicrobial agent derived from Streptomyces roseosporus and it has in vitro bactericidal activity against grampositive pathogens. ${ }^{2,4}$ The clinical experience with daptomycin therapy in pediatric patients

a. Pediatric Infectious Diseases Unit, Department of Pediatrics, Hacettepe University Faculty of Medicine, Ankara, Turkey.

b. Bone Marrow Transplantation Unit, Department of Pediatrics, Hacettepe University Faculty of Medicine, Ankara, Turkey.

c. Department of Medical Microbiology, Hacettepe University Faculty of Medicine, Ankara, Turkey.

E-mail Address:

Ayşe Büyükcam, M.D.: dr.aysebaktir@gmail.com

Funding: None.

Conflict of interest: None.

Received: 3-16-2016

Accepted: 5-23-2016 with VRE is limited. Moreover, drug dosage, safety and tolerability have not been definitely established in children. ${ }^{2}$ We report our experience about treatment of a VRE bacteremia in a 3-yearold boy with acute myeloid leukemia (AML)-M7 and Down syndrome.

\section{CASE REPORT}

A 3-year-old patient with Down syndrome and AML-M7 was admitted to the Emergency Department due to fever at the fourth month of chemotherapy.

Medical history: When he was 23 months old, he was brought to the clinic with bone pain and inability to walk; he was diagnosed with pre B cell leukemia. St Jude's Total XV chemotherapy protocol was given for leukemia. The patient's foot pain repeated at the 28 weeks of chemotherapy; bone marrow aspiration revealed AML-M7. His treatment protocol was switched to AML BFM 2004. His past medical history was also remarkable for congenital heart disease (atrial septal defect and ventricular septal defect).

Physical examination: He was febrile (tympanic temperature was $38.5^{\circ} \mathrm{C}$ ), $2 / 6$ systolic murmur was audible and hepatosplenomegaly was detected.

Laboratory data: Hemoglobin of $11.8 \mathrm{~g} / \mathrm{dL}$, platelet count of $190 \times 10^{3} / \mu \mathrm{L}$ and a white blood cell count of $6700 / \mu \mathrm{L}$ (neutrophils $68 \%$, lymphocytes and blasts $14 \%$, monocytes $18 \%$ ), with normal liver enzymes, kidney function tests and urinalysis. His erythrocyte sedimentation rate was $43 \mathrm{~mm} / \mathrm{h}$ and C-reactive protein was $27.2 \mathrm{mg} / \mathrm{dL}$ (normal: 0-0,8 mg/dL).

The diagnosis of AML relapse was confirmed with bone marrow biopsy examination.

Day 1: The patient was hospitalized and meropenem was started due to neutropenic fever.

Day 7: Teicoplanin, amikacin were empirically added to the treatment because of the persistence of the fever.

Day 8: Liposomal amphotericin B was added.

Day 10: On the following days, pneumonia was diagnosed under antibiotics and trimetroprime sulfamethaxazole started. The antibiotics were 
going on due to the clinical deterioration and prolonged fever. It was known that the patient was colonized with vancomycin-resistant Enterococcus faecium for the last eight months as detected in the surveillance cultures of stool. VRE was isolated from blood cultures which were taken from the central venous port and the peripheral vein on admission (VRE susceptibilities are shown in Table 1).

Day 10: Linezolid (10 mg/ $\mathrm{kg} /$ dose every 8 hours) was added and immunoglobulin M-enriched immunoglobulin (pentaglobulin $4.8 \mathrm{~g} /$ dose) was administered for four times.

Day 12: Ciprofloxacine was added for progression of pneumonia (Figure 1).

Day 13: VRE was still being isolated from blood cultures at the third day of linezolid treatment.
The central venous port was removed and replaced with a new vascular catheter on the $13^{\text {th }}$ day of therapy. Both procedures were also performed at the same time because of the necessity of vascular access. Trans-thoracic echocardiogram did not reveal valve vegetation.

Day 21: Amikacin was switched with gentamycin and repeat blood cultures continued to grow VRE.

Day 22: Daptomycin (8 mg/kg/day, a single dose) was added to linezolid at the $12^{\text {th }}$ day of therapy after consent of the parents and approval by The Ministry of Health of Turkey. Linezolid was continued after the beginning of daptomycin treatment.

Day 32: The blood culture of the patient was sterile. Daptomycin therapy was continued for 5 -weeks during which the blood culture were negative (Table 2).

TABLE 1. VRE, susceptibilities

\begin{tabular}{|c|c|c|c|}
\hline Antimicrobial & $\begin{array}{c}\text { Method of } \\
\text { susceptibility Testing }\end{array}$ & $\begin{array}{c}\text { MIC } \\
(\mu \mathrm{g} / \mathrm{mL})\end{array}$ & $\begin{array}{l}\text { Susceptibility } \\
\text { interpretation }\end{array}$ \\
\hline Ampicillin & Vitek2 system $(\mathrm{V} 2 \mathrm{~S})^{*}$ & $\geq 32$ & $\mathrm{R}$ \\
\hline Imipenem & $\mathrm{V} 2 \mathrm{~S}$ & $\geq 16$ & $\mathrm{R}$ \\
\hline High levels of gentamicin & V2S & SYN-S & S \\
\hline High levels of streptomycin & V2S & SYN-R & $\mathrm{R}$ \\
\hline Ciprofloxacin & V2S & $\geq 8$ & $\mathrm{R}$ \\
\hline Erythromycin & V2S & $\geq 8$ & $\mathrm{R}$ \\
\hline Clindamycin & V2S & $\geq 8$ & $\mathrm{R}$ \\
\hline Linezolid & $\mathrm{V} 2 \mathrm{~S}$ & 2 & S \\
\hline Teicoplanin & V2S & $\geq 32$ & $\mathrm{R}$ \\
\hline Vancomycin & V2S & $\geq 32$ & $\mathrm{R}$ \\
\hline Tetracycline & V2S & $\geq 16$ & $\mathrm{R}$ \\
\hline Tigecycline & V2S & $\leq 12$ & S \\
\hline Trimethoprim Sulfametoxazol & V2S & $\geq 320$ & $\mathrm{R}$ \\
\hline Daptomycin & E-test & 2 & $\mathrm{~S}$ \\
\hline
\end{tabular}

Abbreviations: MIC, minimum inhibitory concentration.

*BioMerieux France.

FIGURE 1. Antimicrobial therapy and blood culture results of patient during daptomycin treatment

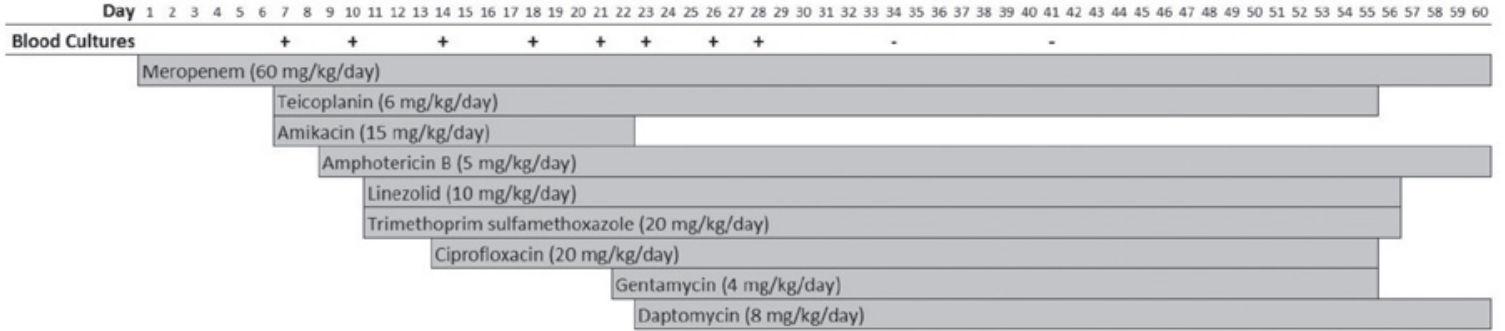


Serum CPK levels were in normal limits during the treatment. On 3th, 9th and 13th days after completion of daptomycin treatment the blood cultures were persistently negative. The previously planned FLAG IDA protocol for relapse AML was given. The patient died at the $77^{\text {th }}$ day of hospitalization due to pulmonary infection, bleeding and progression of AML. No specific microorganism was detected during the last episode of pulmonary infection and progression of disease.

\section{DISCUSSION}

Vancomycin-resistant enterococcal bacteremia has an unfavorable outcome in children. ${ }^{5}$ While the various agents including quinupristindalfopristin, daptomycin, and tigecyline are being used for VRE infections in adults, limited treatment options are significant problems for children. The clinical cure rate of infections with antibiotic resistant gram-positive pathogens was reported as $69.7 \%$ in children treated with linezolid. ${ }^{6}$ In another study by Grazzino et al., the clinical cure or improvement of VRE infections with linezolid was achieved in $66.7 \%$ of the pediatric patients. ${ }^{7}$ However, linezolid has bacteriostatic activity to enterococcci and treatment failure may be seen despite susceptibility of VRE and removal of central venous port as in our patient. ${ }^{8}$ Daptomycin has appeared as another alternative for the treatment of VRE infections in pediatric population in recent years. The effectiveness of the treatment was shown in several reports in children with meningitis, bacteremia, sepsis, endocarditis, and urinary tract infections caused by VRE. ${ }^{2,9,10} \mathrm{~A}$ certain number of combination therapy including ampicilline, aminogycosides, ceftalorine, tigecyline, rifampicin, and doxycycline with daptomycine has been used succesfully for VRE infections in the literature ${ }^{3}$. In addition synergy between daptomycin and gentamicin, for highlevel gentamicin susceptible E. faecium has been already known ${ }^{11}$ and it occured in our treatment. Therefore, we cannot generalize the potential benefit of multiantimicrobial usage.

Data are limited on the usage of daptomycin for pediatric patients. Also effective doses of daptomycin in children are different from adults and the optimal dosage is not well established. Daptomycin mostly binds a protein in circulation and is eliminated through kidneys. ${ }^{2}$ Limited pharmacokinetic studies have manifested faster daptomycin clearance in children and it seems to have a shorter half-life in children aged 2-6 when compared to children aged $12-17 .{ }^{10}$ Higher doses may be required in younger patients and the administration of $15 \mathrm{mg} / \mathrm{kg} / 12 \mathrm{~h}$ was reported as a maximum dose in the literature in an infant. ${ }^{2,8}$ Our patient was treated at a dose of $8 \mathrm{mg} / \mathrm{kg} /$

TABLE 2. The patient's cultures during the treatment

\begin{tabular}{|c|c|c|c|}
\hline \multirow[t]{2}{*}{ Treatment day } & \multicolumn{2}{|c|}{ Blood culture } & \multirow[t]{2}{*}{ Other cultures } \\
\hline & Central venous port/vasculer catheter* & Peripheral vein & \\
\hline 7 th & $\operatorname{VRE}(+)$ & VRE (+) & \\
\hline 8 th & & & Urine (-) \\
\hline 10 th & $\operatorname{VRE}(+)$ & $\operatorname{VRE}(+)$ & \\
\hline 14 th & $\operatorname{VRE}(+)$ & VRE (+) & \\
\hline 18 th & $\operatorname{VRE}(+)$ & $\operatorname{VRE}(+)$ & \\
\hline 21 th & $\operatorname{VRE}(+)$ & & \\
\hline 23 th & & $\operatorname{VRE}(+)$ & \\
\hline 26 th & $\operatorname{VRE}(+)$ & $\operatorname{VRE}(+)$ & \\
\hline 27 th & & & Pleural fluide (-) \\
\hline 28 th & $\operatorname{VRE}(+)$ & $\operatorname{VRE}(+)$ & \\
\hline 33th & & & Pleural fluide (-) \\
\hline 34 th & - & - & \\
\hline 41th & - & & \\
\hline 63 th $^{* *}$ & - & - & \\
\hline
\end{tabular}

* Port was removed and replaced with a new vascular catheter on the $13^{\text {th }}$ of therapy.

**Cultures were taken after three days the discontinuation of daptomycin. 
day and the use of daptomycin resulting in microbiological and clinical cure of infection without the observation of any side effects.

Several factors have related to an increase in the incidence of VRE infection, which may be attributed to several factors that increase the risk of infection, such as colonization of gastrointestinal tract, prior vancomycin or fluoroquinolone therapy, higher Acute Physiology and Chronic Health Evaluation II (APACHE) score and previous corticosteroid usage. Furthermore, in many reports, underlying hematologic malignancy, receiving chemotherapy, and high-grade bacteremia have been reported; the isolation of VRE from blood is significantly correlated with mortality. However these risk factors were showed in adult population and we have insufficient data in pediatric patients. ${ }^{12,13}$ The present case of persistent bloodstream VRE infection occurred in a boy with severe neutropenia following chemotherapy for relapsed AML-M7, and its clinical course shared similarities with those previously reported, particularly the poor outcome.

\section{CONCLUSION}

This report highlights the therapeutic approach of the persistent VRE bacteremia in a child with AML-M7 and Down syndrome, treated successfully with daptomycin. Despite susceptibility to linezolid, modification of antimicrobial therapy to daptomycin resolved the fever with favorable clinical and microbiological response in this case. We thought that more studies are needed to clarify the pharmacodynamics, pharmacokinetics, tolerability and toxicity of daptomycin in pediatric population, especially patients with underlying chronic diseases.

\section{REFERENCES}

1. Tamma PD, Hsu AJ. Optimizing therapy for vancomycinresistant enterococcal bacteremia in children. Curr Opin Infect Dis 2014;27(6):517-27.

2. Principi N, Caironi M, Venturini F, Pani L, etal. Daptomycin in paediatrics: current knowledge and the need for future research. J Antimicrob Chemother 2015 Mar;70(3):643-8.

3. Munita JM, Murray BE, Arias CA. Daptomycin for the treatment of bacteraemia due to vancomycin-resistant enterococci. Int J Antimicrob Agents 2014;44(5):387-95.

4. Humphries RM,PollettS, Sakoulas G. A current perspective on daptomycin for the clinical microbiologist. Clin Microbiol Rev 2013;26(4):759-80.

5. Diaz Granados CA, Zimmer SM, Klein M, Jernigan JA. Comparison of mortality associated with vancomycinresistant and vancomycin-susceptible enterococcal bloodstream infections: a meta-analysis. Clin Infect Dis 2005;41(3):327-33.

6. Jantausch BA, Deville J, AdlerS, Morfin MR, etal. Linezolid for the treatment of children with bacteremia or nosocomial pneumonia caused by resistant gram-positive bacterial pathogens. Pediatr Infect Dis J 2003;22(9 Suppl):S164-71.

7. GarazzinoS, Tovo PA. Clinical experience with linezolid in infants and children. J Antimicrob Chemother 2011;66 Suppl 4:iv23-iv41.

8. Beneri CA, Nicolau DP, Seiden HS, Rubin LG. Successful treatment of a neonate with persistent vancomycin-resistant enterococcal bacteremia with a daptomycin-containing regimen. Infect Drug Resist 2008;1:9-11.

9. Ardura MI, Mejias A, Katz KS, Revell P, et al. Daptomycin therapy for invasive Gram-positive bacterial infections in children. Pediatr Infect Dis J 2007;26(12):1128-32.

10. Durand C, Brueckner A, Sampadian C, Willett KC, et al. Daptomycin use in pediatric patients. Am J Health Syst Pharm 2014;71(14):1177-82.

11. Hindler JA, Wong-Beringer A, Charlton CL, Miller SA, et al. In vitro activity of daptomycin in combination with beta-lactams, gentamicin, rifampin and tigecycline against daptomycin-nonsusceptibleenterococci. Antimicrob Agents Chemother 2015;59(7):4279-88.

12. Ornstein MC, MukherjeeS, Keng M, Elson P, et al. Impact of vancomycin-resistant enterococcal bacteremia on outcome during acute myeloid leukemia induction therapy. Leuk Lymphoma 2015;56(9):2536-42.

13. KraftS, Mackler E, Schlickman P, Welch K, et al. Outcomes of therapy: vancomycin-resistantenterococcal bacteremia in hematology and bone marrow transplant patients. Support Care Cancer 2011;19(12):1969-74. 